PROCEEDINGS OF THE

AMERICAN MATHEMATICAL SOCIETY

Volume 136, Number 12, December 2008, Pages 4267-4273

S 0002-9939(08)09631-7

Article electronically published on July 16, 2008

\title{
ISOMETRIC WEIGHTED COMPOSITION OPERATORS ON WEIGHTED BANACH SPACES OF TYPE $H^{\infty}$
}

\author{
JOSÉ BONET, MIKAEL LINDSTRÖM, AND ELKE WOLF
}

(Communicated by N. Tomczak-Jaegermann)

\begin{abstract}
We characterize those weighted composition operators on weighted Banach spaces of holomorphic functions of type $H^{\infty}$ which are an isometry.
\end{abstract}

\section{INTRODUCTION}

Let $v$ be a strictly positive continuous function (weight) on the open unit disk $D$ in the complex plane which is radial (that is, $v(z)=v(|z|)$ for every $z \in D$ ) and decreasing with respect to $|z|$. We are interested in operators defined on the weighted Banach spaces of holomorphic functions

$$
H_{v}^{\infty}:=\left\{f \in H(D) ;\|f\|_{v}=\sup _{z \in D} v(z)|f(z)|<\infty\right\}
$$

and on the smaller spaces

$$
H_{v}^{0}:=\left\{f \in H_{v}^{\infty}, \lim _{|z| \rightarrow 1} v(z)|f(z)|=0\right\},
$$

both endowed with the norm $\|\cdot\|_{v}$. Here $H(D)$ denotes the space of all analytic functions. Spaces of this type appear in the study of growth conditions of analytic functions and have been investigated in various articles; see e.g. [10, 11, 14, [13, 2], 3, 4. We consider non-constant functions $\varphi \in H(D)$ satisfying $\varphi(D) \subset D$ as well as functions $\psi \in H(D)$ which are not identically equal to zero. These functions induce the weighted composition operator $C_{\varphi, \psi}$ which is a linear map on $H(D)$ and given by $C_{\varphi, \psi}=\psi(f \circ \varphi)$. If $\psi=1$, then as usual we denote $C_{\varphi, 1}$ by $C_{\varphi}$.

In 16. M.J. Martin and D. Vukotic analyze when composition operators on the Bloch space are a (not necessarily surjective) isometry, and they show that every thin Blaschke product induces an isometric composition operator on the Bloch space. The same authors give a unified proof of the characterization of all possible composition operators that are isometries on some classical function spaces; see [15]. Laitila [1] characterizes analytic self-maps $\varphi$ which induce isometric composition operators on the space BMOA equipped with a Möbius invariant $H^{2}$-norm. A good general reference on isometries of spaces of analytic functions is the book of R. Fleming and J. Jamison [8].

Received by the editors August 6, 2007.

2000 Mathematics Subject Classification. Primary 47B33, 47B38.

Key words and phrases. Isometry, weighted composition operator, weighted Banach space of holomorphic functions. 
In this paper our aim is to characterize isometric weighted composition operators on weighted Banach spaces of holomorphic functions as defined above, thus extending the result of M.J. Martin and D. Vukotic in [16]. Our main results are Theorem 2 and Theorem 9 .

\section{NOTATION AND PRELIMINARIES}

For notation and more information on composition operators we refer the reader to the excellent monographs [6] and [19]. In order to formulate results on weighted spaces of analytic functions we need the so-called associated weights (see [2]). For a weight $v$ the associated weight $\tilde{v}$ is given by

$$
\tilde{v}(z):=\frac{1}{\sup \left\{|f(z)| ; f \in H_{v}^{\infty},\|f\|_{v} \leq 1\right\}}=\frac{1}{\left\|\delta_{z}\right\|_{H_{v}^{\infty}}}, z \in D,
$$

where $\delta_{z}$ denotes the point evaluation of z. By [2] we know that associated weights are continuous, $\tilde{v} \geq v>0$ and that for each $z \in D$ we can find $f_{z} \in H_{v}^{\infty},\|f\|_{v} \leq 1$, such that $\left|f_{z}(z)\right|=\frac{1}{\tilde{v}(z)}$. It is well known that $H_{\tilde{v}}^{\infty}$ is isometrically isomorphic to $H_{v}^{\infty}$. We are especially interested in radial weights which satisfy the following condition, which is due to W. Lusky (see [13]):

$$
\inf _{k} \frac{v\left(1-2^{-k-1}\right)}{v\left(1-2^{-k}\right)}>0 \text {. }
$$

The standard weights $v_{p}(z)=\left(1-|z|^{2}\right)^{p}, p>0$, are weights which have $(L 1)$.

We also need some geometric data of the unit disk. The pseudohyperbolic metric is given by

$$
\rho(z, a):=\left|\sigma_{a}(z)\right|, \text { where } \sigma_{a}(z):=\frac{a-z}{1-\bar{a} z}
$$

is the automorphism of $\mathbb{D}$ which changes 0 and $a$. Furthermore, we will use the following equality, which is easy to check:

$$
1-\left|\sigma_{a}(z)\right|^{2}=\frac{\left(1-|a|^{2}\right)\left(1-|z|^{2}\right)}{|1-\bar{a} z|^{2}} .
$$

\section{ISOMETRIC WEIGHTED COMPOSITION OPERATORS}

The surjective isometries on the spaces $H_{v_{p}}^{\infty}, 0<p<\infty$, can be characterized following the argument given by J. Cima and W. Wogen in [7], 8] for $H_{v_{1}}^{\infty}$. More precisely, if $S: H_{v_{p}}^{\infty} \rightarrow H_{v_{p}}^{\infty}$ is a surjective isometry, then there is a conformal automorphism $\varphi$ of $D$ and $\lambda \in \partial D$ such that $S f=C_{\varphi, \lambda\left(\varphi^{\prime}\right)^{p}} f$ for every $f \in H_{v_{p}}^{\infty}$. Even for the standard weights a characterization of all isometries on these spaces does not seem to be known. But in the case of the smaller space $H_{v_{p}}^{0}$ all isometries can be described in this way (see [7, 8]). Therefore we will focus our attention on isometries among the weighted composition operators acting on $H_{v}^{\infty}$ for more general weights $v$.

The following result, which is crucial for the proof of our main result, can be found in [12] (see [9, Lemma 5.1] for the case of standard weights $v_{p}$ ).

Lemma 1. Let $v$ be a radial weight with $(L 1)$ such that $v$ is continuously differentiable with respect to $|z|$. Then there is $M>0$ such that for $f \in H_{v}^{\infty}$ we have

$$
|v(p) f(p)-v(q) f(q)| \leq M\|f\|_{v} \rho(p, q)
$$

for all $p, q \in D$. 
Theorem 2. Let $\varphi$ be an analytic self-map of $D$ and $\psi \in H(D)$.

(a) Assume that $v$ is a radial weight with (L1) such that $v$ is continuously differentiable with respect to $|z|$. If $\sup _{z \in D} \frac{|\psi(z)| v(z)}{\tilde{v}(\varphi(z))} \leq 1$ and the following condition holds:

$\left(M_{\varphi, \psi, v}\right) \quad$ for every $a \in D$ there exists a sequence $\left(z_{n}\right)_{n} \subset D$ such that

$$
\rho\left(\varphi\left(z_{n}\right), a\right) \rightarrow 0 \text { and } \frac{\left|\psi\left(z_{n}\right)\right| v\left(z_{n}\right)}{\tilde{v}\left(\varphi\left(z_{n}\right)\right)} \rightarrow 1,
$$

then $C_{\varphi, \psi}: H_{v}^{\infty} \rightarrow H_{v}^{\infty}$ is an isometry.

(b) Let $v$ be a radial weight such that $w(z):=\frac{v(z)}{\left(1-|z|^{2}\right)^{p}}$ is a weight on $D$ for some $0<p<\infty$ and $w=\tilde{w}$. If $C_{\varphi, \psi}: H_{v}^{\infty} \rightarrow H_{v}^{\infty}$ is an isometry, then condition $\left(M_{\varphi, \psi, v}\right)$ is satisfied and $\sup _{z \in D} \frac{|\psi(z)| v(z)}{\tilde{v}(\varphi(z))} \leq 1$.

Proof. (a) By assumption, for each $f \in H_{v}^{\infty}$ we have that

$$
\left\|C_{\varphi, \psi} f\right\|_{v}=\sup _{z \in D} \frac{|\psi(z)| v(z)}{\tilde{v}(\varphi(z))} \tilde{v}(\varphi(z))|f(\varphi(z))| \leq\|f\|_{v} .
$$

In order to verify the reverse inequality, let $f \in H_{v}^{\infty}$. Then

$$
\|f\|_{v}=\lim _{m \rightarrow \infty} v\left(a_{m}\right)\left|f\left(a_{m}\right)\right|
$$

for some sequence $\left(a_{m}\right)_{m}$. Let $m$ be fixed. Hence, by condition $\left(M_{\varphi, \psi, v}\right)$, there is a sequence $\left(z_{n}^{m}\right)_{n} \subset D$ such that $\rho\left(\varphi\left(z_{n}^{m}\right), a_{m}\right) \rightarrow 0$ and $\frac{\left|\psi\left(z_{n}^{m}\right)\right| v\left(z_{n}^{m}\right)}{\tilde{v}\left(\varphi\left(z_{n}^{m}\right)\right)} \rightarrow 1$, when $n \rightarrow \infty$. By Lemma 1 , for all $m$ and $n$,

$$
\left|v\left(a_{m}\right) f\left(a_{m}\right)-v\left(\varphi\left(z_{n}^{m}\right)\right) f\left(\varphi\left(z_{n}^{m}\right)\right)\right| \leq M\|f\|_{v} \rho\left(a_{m}, \varphi\left(z_{n}^{m}\right)\right) .
$$

Hence

$$
\begin{gathered}
\left\|C_{\varphi, \psi} f\right\|_{v}=\sup _{z \in D} \frac{|\psi(z)| v(z)}{\tilde{v}(\varphi(z))} \tilde{v}(\varphi(z))|f(\varphi(z))| \\
\geq \limsup _{n \rightarrow \infty} \frac{\left|\psi\left(z_{n}^{m}\right)\right| v\left(z_{n}^{m}\right)}{\tilde{v}\left(\varphi\left(z_{n}^{m}\right)\right)}\left(\left|f\left(a_{m}\right)\right| v\left(a_{m}\right)-M\|f\|_{v} \rho\left(\varphi\left(z_{n}^{m}\right), a_{m}\right)\right)=v\left(a_{m}\right)\left|f\left(a_{m}\right)\right| .
\end{gathered}
$$

Since this is true for all $m,\left\|C_{\varphi, \psi} f\right\|_{v} \geq\|f\|_{v}$.

(b) Choose $p>0$ such that $w(z):=\frac{v(z)}{\left(1-|z|^{2}\right)^{p}}$ is a weight on $D$ with $\tilde{w}=w$. First we want to show that this implies $v=\tilde{v}$. Since $v(z) \leq \tilde{v}(z)$ for each $z \in D$, it is enough to show the reverse inequality. Fix $a \in D$, and select $k_{a} \in H(D)$ with $\left|k_{a}\right| \leq 1 / w$ and $\left|k_{a}(a)\right|=1 / w(a)$. If $a \neq 0$, select $b$ with $|b|=1$ and $a=|a| b$ and define $h_{a}(z):=k_{a}(z) /\left(1-(\bar{b} z)^{2}\right)^{p}, z \in D$. Clearly $\left|h_{a}\right| \leq 1 / \tilde{v}$ on $D$, and $\left|h_{a}(a)\right|=1 / v(a)$; thus $1 / v(a) \leq 1 / \tilde{v}(a)$, as desired. In case $a=0$, it is enough to take $h_{0}(z):=k_{0}(z), z \in D$, to reach the conclusion.

By assumption, $\left\|C_{\varphi, \psi} f\right\|_{v}=\|f\|_{v}$ for all $f \in H_{v}^{\infty}$. Hence

$$
\left\|C_{\varphi, \psi}\right\|=\sup _{z \in D} \frac{|\psi(z)| v(z)}{\tilde{v}(\varphi(z))} \leq 1
$$

Let $a \in D$. Then there exists $g_{a} \in H_{w}^{\infty}(D),\left\|g_{a}\right\|_{w}=1$ with $g_{a}(a) \tilde{w}(a)=1$. Put

$$
f_{a}(z)=g_{a}(z)\left(\frac{1-|a|^{2}}{(1-z \bar{a})^{2}}\right)^{p} .
$$


It follows that $\left\|f_{a}\right\|_{v}=1$ since $\left|f_{a}(a)\right| v(a)=1$. Thus we can pick a sequence $\left(z_{n}\right)_{n} \subset D$ so that $\left|\psi\left(z_{n}\right)\right|\left|f_{a}\left(\varphi\left(z_{n}\right)\right)\right| v\left(z_{n}\right) \rightarrow 1$ when $n \rightarrow \infty$.

Hence

$$
1 \geq \frac{\left|\psi\left(z_{n}\right)\right| v\left(z_{n}\right)}{\tilde{v}\left(\varphi\left(z_{n}\right)\right)} \geq \frac{\left|\psi\left(z_{n}\right)\right| v\left(z_{n}\right)}{\tilde{v}\left(\varphi\left(z_{n}\right)\right)}\left|f_{a}\left(\varphi\left(z_{n}\right)\right)\right| \tilde{v}\left(\varphi\left(z_{n}\right)\right)=\left|\psi\left(z_{n}\right)\right| v\left(z_{n}\right)\left|f_{a}\left(\varphi\left(z_{n}\right)\right)\right|,
$$

SO

Further,

$$
\lim _{n \rightarrow \infty} \frac{\left|\psi\left(z_{n}\right)\right| v\left(z_{n}\right)}{\tilde{v}\left(\varphi\left(z_{n}\right)\right)}=1 .
$$

$$
\begin{gathered}
1 \geq\left(1-\left|\sigma_{a}\left(\varphi\left(z_{n}\right)\right)\right|^{2}\right)^{p}=\frac{\left(1-|a|^{2}\right)^{p}\left(1-\left|\varphi\left(z_{n}\right)\right|^{2}\right)^{p}}{\left|1-\varphi\left(z_{n}\right) \bar{a}\right|^{2 p}} \\
=\frac{\left|f_{a}\left(\varphi\left(z_{n}\right)\right)\right| v\left(\varphi\left(z_{n}\right)\right)\left(1-\left|\varphi\left(z_{n}\right)\right|^{2}\right)^{p}}{\left|g_{a}\left(\varphi\left(z_{n}\right)\right)\right| v\left(\varphi\left(z_{n}\right)\right)} \geq\left|f_{a}\left(\varphi\left(z_{n}\right)\right)\right| v\left(\varphi\left(z_{n}\right)\right) .
\end{gathered}
$$

Since $\left|f_{a}\left(\varphi\left(z_{n}\right)\right)\right| \tilde{v}\left(\varphi\left(z_{n}\right)\right) \rightarrow 1$, when $n \rightarrow \infty$, we conclude, as $v=\tilde{v}$, that $\lim _{n \rightarrow \infty}\left(1-\left|\sigma_{a}\left(\varphi\left(z_{n}\right)\right)\right|^{2}\right)^{p}=1$ and $\rho\left(\varphi\left(z_{n}\right), a\right) \rightarrow 0$ when $n \rightarrow \infty$.

Example 3. Besides the standard weights $v_{p}$ the following weights also satisfy the assumptions in Theorem 2 (b):

$$
v(z)=\left(1-|z|^{2}\right)^{p}\left(1-\log \left(1-|z|^{2}\right)\right)^{-\beta}, \quad 0<p<\infty \text { and } \beta>0 .
$$

Example 4. (i) Fix $b \in D$ and $0<p<\infty$. Put $\varphi(z):=\sigma_{b}(z)$ and $\psi(z):=$ $\left(\sigma_{b}^{\prime}\right)^{p}(z)$. Then an easy calculation shows that $C_{\varphi, \psi}: H_{v_{p}}^{\infty} \rightarrow H_{v_{p}}^{\infty}$ satisfies condition $\left(M_{\varphi, \psi, v_{p}}\right)$ as well as $\sup _{z \in D} \frac{|\psi(z)| v_{p}(z)}{v_{p}(\varphi(z))} \leq 1$, so by Theorem 2 it is an isometry, but 0 is not a fixed point of the self-map.

(ii) Analogously to the proof of [16, Theorem 1.2] we can show that for each thin Blaschke product $B$ such that $B(0)=0$ the weighted composition operator $C_{B,\left(B^{\prime}\right)^{p}}: H_{v_{p}}^{\infty} \rightarrow H_{v_{p}}^{\infty}$ is an isometry for every $0<p<\infty$.

Let $B_{p}, 0<p<\infty$, denote the Bloch type spaces of functions $f \in H(D)$ with $f(0)=0$ satisfying $\|f\|_{p}:=\sup _{z \in D}\left(1-|z|^{2}\right)^{p}\left|f^{\prime}(z)\right|<\infty$. Then $\|\cdot\|_{p}$ becomes a norm and $B_{p}$ a Banach space. The map $S_{p}: B_{p} \rightarrow H_{v_{p}}^{\infty}$ given by $S_{p}(f)=f^{\prime}$ is a surjective isometry.

Since $C_{\varphi}=S_{p}^{-1} \circ C_{\varphi, \varphi^{\prime}} \circ S_{p}$, we have that $C_{\varphi}: B_{p} \rightarrow B_{p}$ is an isometry if and only if $C_{\varphi, \varphi^{\prime}}: H_{v_{p}}^{\infty} \rightarrow H_{v_{p}}^{\infty}$ is as well. By Theorem 2 we get the following result for Bloch type spaces.

Corollary 5. Let $\varphi$ be an analytic self-map of $D$ and $0<p<\infty$. The operator $C_{\varphi}: B_{p} \rightarrow B_{p}$ is an isometry if and only if $\sup _{z \in D} \frac{\left|\varphi^{\prime}(z)\right| v_{p}(z)}{v_{p}(\varphi(z))} \leq 1$ and the following condition holds:

$\left(M_{\varphi, \varphi^{\prime}, v_{p}}\right) \quad$ for every $a \in D$ there exists a sequence $\left(z_{n}\right)_{n} \subset D$ such that

$$
\rho\left(\varphi\left(z_{n}\right), a\right) \rightarrow 0 \text { and } \frac{\left|\varphi^{\prime}\left(z_{n}\right)\right| v_{p}\left(z_{n}\right)}{v_{p}\left(\varphi\left(z_{n}\right)\right)} \rightarrow 1 .
$$

Let $\mathcal{B}$ denote the classical Bloch space of all analytic functions $f$ with the norm $\|f\|_{\mathcal{B}}=|f(0)|+\|f\|_{1}<\infty$. If $\varphi(0)=0$, then clearly $C_{\varphi}: \mathcal{B} \rightarrow \mathcal{B}$ is an isometry if and only if $C_{\varphi}: B_{1} \rightarrow B_{1}$ is an isometry. From $\left\|C_{\varphi} f\right\|_{\mathcal{B}}=\|f\|_{\mathcal{B}}$ for all $f \in \mathcal{B}$, it follows that $|f(\varphi(0))| \geq|f(0)|$ for all $f \in \mathcal{B}$. By choosing $f(z):=\frac{\varphi(0)-z}{1-\overline{\varphi(0)} z} \in \mathcal{B}$, one gets $\varphi(0)=0$ (see [15]). Therefore we obtain Theorem 1.1 in [16]. 
Corollary 6. Let $\varphi$ be an analytic self-map of $D$. Then $C_{\varphi}: \mathcal{B} \rightarrow \mathcal{B}$ is an isometry if and only if $\varphi(0)=0$ and the following condition holds:

$\left(M_{\varphi, \varphi^{\prime}, v_{1}}\right) \quad$ for every $a \in D$ there exists a sequence $\left(z_{n}\right)_{n} \subset D$ such that

$$
\rho\left(\varphi\left(z_{n}\right), a\right) \rightarrow 0 \text { and } \frac{\left|\varphi^{\prime}\left(z_{n}\right)\right|\left(1-\left|z_{n}\right|^{2}\right)}{1-\left|\varphi\left(z_{n}\right)\right|^{2}} \rightarrow 1 .
$$

Now we restrict our attention to (non-weighted) composition operators, and obtain a result that should be compared with M.J. Martin, D. Vukotic [15, Theorem 1.3(b)].

If $v$ is radial there are only two possibilities: Either $\tilde{v}(r)$ tends to 0 as $r$ tends to 1 or it tends to a positive number. In the second case $H_{v}^{\infty}=H^{\infty}$. This is why we consider first the case $H^{\infty}$, which is essentially known. Let $S$ be any isometry of $H^{\infty}$ onto $H^{\infty}$. Then $S$ has the form $S f=\lambda C_{\varphi} f$ for every $f \in H^{\infty}$, where $\varphi$ is a conformal automorphism of $D$ and $\lambda \in \partial D$ (see [8, Theorem 4.2.2]). In [17] R. Roan shows that $C_{\varphi}: H^{\infty} \rightarrow H^{\infty}$ has closed range if and only if $\partial D \subset \overline{\varphi(D)}$. His argument also characterizes isometry of $C_{\varphi}$ on $H^{\infty}$. Indeed, by using Lemma 3 in D. Sarason [18, one concludes that $\partial D \subset \overline{\varphi(D)}$ implies

$$
\left\{z \in D ;|f(z)| \leq \sup _{w \in \varphi(D)}|f(w)| \forall f \in H^{\infty}\right\}=D .
$$

Thus, for $f \in H^{\infty}$,

$$
\|f\|_{\infty} \geq\left\|C_{\varphi} f\right\|_{\infty}=\sup _{z \in D}|f(\varphi(z))| \geq\|f\|_{\infty} .
$$

Theorem 7. Let $\varphi: D \rightarrow D$ be an analytic self-map. The following statements are equivalent:

(i) $C_{\varphi}: H^{\infty} \rightarrow H^{\infty}$ is an isometry.

(ii) $C_{\varphi}: H^{\infty} \rightarrow H^{\infty}$ has closed range.

(iii) $\partial D \subset \overline{\varphi(D)}$.

Example 8. By [8] $\varphi: D \rightarrow D, \varphi(z)=\frac{-1+2(1+i z)}{1+i z+\sqrt{2\left(1-z^{2}\right)}}$ is not a surjection but satisfies $\overline{\varphi(D)}=\bar{D}$, and therefore $C_{\varphi}$ is an isometry on $H^{\infty}$.

We treat now isometric composition operators on $H_{v}^{\infty}$ when the weight $v$ satisfies that $\tilde{v}(r)$ tends to 0 as $r$ tends to 1 .

Theorem 9. Let $v$ be a radial weight such that $\lim _{r \rightarrow 1} \tilde{v}(r)=0$. Let $\varphi$ be an analytic self-map on $D$ such that $\varphi(0)=0$. The composition operator $C_{\varphi}$ on $H_{v}^{\infty}$ is an isometry if and only if $\varphi$ is a rotation.

Proof. If $\varphi$ is a rotation, then $C_{\varphi}$ is an isometry, since $v$ is radial. Conversely, since $\varphi(0)=0$, the Schwarz lemma gives $|\varphi(z)| \leq|z|$ for each $z \in D$. Set $\tilde{v}(1):=0$. Recall that $\tilde{v}$ is radial and continuous. It is enough to find $a \in D, a \neq 0$, with $|\varphi(a)|=|a|$. To do this, find $b \in] 0,1\left[\right.$ with $\tilde{v}(b) b=\max _{0 \leq r \leq 1} r \tilde{v}(r)$, and $\tilde{v}(z)|z|<\tilde{v}(b) b$ if $b<|z|<1$. Select $b<c<1$ and find $d \in\left[c, 1\left[\right.\right.$ with $d \tilde{v}(d)=\max _{c \leq x \leq 1} x \tilde{v}(x)$. Clearly $d \tilde{v}(d)<b \tilde{v}(b)$, by the selection of $b$. For $|z| \geq c$, we have $\tilde{v}(z)|\varphi(z)| \leq$ $\tilde{v}(z)|z| \leq \tilde{v}(d) d<\tilde{v}(b) b$. Since $C_{\varphi}$ is an isometry, $\sup _{z \in D} \tilde{v}(z)|\varphi(z)|=\tilde{v}(b) b$. Find a sequence $\left(z_{j}\right)_{j}$ in $D$ such that $\left|z_{j}\right| \leq c$ for all $j$ and $\tilde{v}\left(z_{j}\right)\left|\varphi\left(z_{j}\right)\right|$ tends to $\tilde{v}(b) b$. Consequently, the sequence has an accumulation point $a$ with $|a| \leq c$. This yields

$$
\sup _{z \in D} \tilde{v}(z)|\varphi(z)|=\tilde{v}(a)|\varphi(a)|=\tilde{v}(b) b=\sup _{z \in D} \tilde{v}(z)|z|>0 .
$$


In particular $a \neq 0$, since otherwise $\varphi(a)=0$. This implies $\tilde{v}(a)|\varphi(a)| \geq \tilde{v}(a)|a|$; hence $|\varphi(a)|=|a|$. The proof is complete.

Proposition 10. Let $v$ be a radial weight such that its associated weight $\tilde{v}$ is strictly decreasing on $[0,1[$. Let $\varphi$ be an analytic self-map of D. If the composition operator $C_{\varphi}$ is an isometry on $H_{v}^{\infty}(D)$, then $\varphi(0)=0$.

Proof. Recall that $H_{v}^{\infty}$ and $H_{\tilde{v}}^{\infty}$ coincide isometrically. Fix $a \in D$. Select $f \in H_{v}^{\infty}$ such that $|f| \leq 1 / v,|f(\varphi(a))|=1 / \tilde{v}(\varphi(a))$. Clearly $\|f\|_{v}=1=\tilde{v}(\varphi(a))|f(\varphi(a))|$. This implies

$$
1=\left\|C_{\varphi}(f)\right\|_{v} \geq \tilde{v}(a)|f(\varphi(a))|=\tilde{v}(a) / \tilde{v}(\varphi(a)) .
$$

This yields $\tilde{v}(a) \leq \tilde{v}(\varphi(a))$, and since $\tilde{v}$ is strictly decreasing, $|\varphi(a)| \leq|a|$. Applying this for $a=0$, we conclude $\varphi(0)=0$.

Remark 11. Let $\varphi$ be an analytic self-map of $D$ and $0<p<\infty$. Then $\varphi$ is a rotation if and only if $\varphi(0)=0$ and the following property is valid:

$\left(M_{\varphi, 1, v_{p}}\right) \quad$ for every $a \in D$ there exists a sequence $\left(z_{n}\right)_{n} \subset D$ such that

$$
\rho\left(\varphi\left(z_{n}\right), a\right) \rightarrow 0 \text { and } \frac{v_{p}\left(z_{n}\right)}{v_{p}\left(\varphi\left(z_{n}\right)\right)} \rightarrow 1 .
$$

Indeed, suppose that $\varphi(0)=0$ and $\left(M_{\varphi, 1, v_{p}}\right)$ holds. Then it is enough to prove that there is $b \in D, b \neq 0$, with $|\varphi(b)|=|b|$. Let $a \neq 0, a \in D$. By assumption we can find a sequence $\left(z_{n}\right)_{n} \subset D$ such that $\rho\left(\varphi\left(z_{n}\right), a\right) \rightarrow 0$ and $\frac{v_{p}\left(z_{n}\right)}{v_{p}\left(\varphi\left(z_{n}\right)\right)} \rightarrow 1$. Hence $\left|z_{n}\right| \rightarrow|a|$, when $n \rightarrow \infty$. Now there exists a subsequence $\left(z_{m}\right)$ of $\left(z_{n}\right)_{n}$ such that $z_{m} \rightarrow b$ with $|b| \leq 1$. Therefore $|a|=|b|<1$ and

$$
\|\varphi(b)|-| b\|=|| \varphi(b)|-| a|| \leq 2 \rho(\varphi(b), a)=2 \lim _{m} \rho\left(\varphi\left(z_{m}\right), a\right)=0,
$$

from which we conclude that $|\varphi(b)|=|b|$. The other direction is obvious.

Our last result is a consequence of Theorem 9 and Proposition 10.

Corollary 12. Let $\varphi$ be an analytic self-map on $\mathbb{D}$ and $p>0$. The composition operator $C_{\varphi}$ on $H_{v_{p}}^{\infty}$ is an isometry if and only if $\varphi$ is a rotation.

\section{ACKNOWLEDGEMENT}

The research of J. Bonet was partially supported by MEC-FEDER projects MTM2004-02262 and MTM2007-62643 and the research net MTM2006-26627-E.

\section{REFERENCES}

[1] K.D. Bierstedt, W.H. Summers, Biduals of weighted Banach spaces of analytic functions, J. Austral. Math. Soc. (Series A) 54 (1993), 70-79. MR.1195659 (94a:46027)

[2] K.D. Bierstedt, J. Bonet, J. Taskinen, Associated weights and spaces of holomorphic functions, Studia Math. 127 (1998), 137-168. MR.1488148 (99a:46037)

[3] J. Bonet, P. Domański, M. Lindström, Essential norm and weak compactness of composition operators on weighted Banach spaces of analytic functions, Canad. Math. Bull. 42 (1999), no. 2, 139-148. MR.1692002 (2000d:47052)

[4] J. Bonet, P. Domański, M. Lindström, J. Taskinen, Composition operators between weighted Banach spaces of analytic functions, J. Austral. Math. Soc. (Series A) 64 (1998), 101-118. MR 1490150 (98m:47039)

[5] M.D. Contreras, A.G. Hernández-Díaz, Weighted composition operators in weighted Banach spaces of analytic functions, J. Austral. Math. Soc. (Series A) 69 (2000), 41-60. MR $\overline{1767392}$ $(2001 \mathrm{j}: 47027)$ 
[6] C. Cowen, B. MacCluer, Composition Operators on Spaces of Analytic Functions, CRC Press, Boca Raton, FL, 1995. MR1397026 (97i:47056)

[7] J.A. Cima, W.R. Wogen, On isometries of the Bloch space, Illinois J. Math. 24 (1980), no. 2, 313-316. MR.575069 (82m:30052)

[8] R.J. Fleming, J.E. Jamison, Isometries on Banach Spaces, Chapman and Hall/CRC Monographs and Surveys in Pure and Applied Mathematics, 129, 2003. MR.1957004 (2004j:46030)

[9] H. Hedenmalm, B. Korenblum, K. Zhu, Theory of Bergman Spaces, Graduate Texts in Mathematics, 199, Springer-Verlag, 2000. MR1758653 (2001c:46043)

[10] W. Kaballo, Lifting-Probleme für $H^{\infty}$-Funktionen, Arch. Math. (Basel) 34 (1980), 540-549. MR:596863 (82m:46048)

[11] J. Laitila, Isometric composition operators on BMOA, preprint Helsinki, 2007.

[12] M. Lindström, E. Wolf, Essential norm of the difference of weighted composition operators, Monatshefte für Mathematik 153 (2008), 133-143. MR2373366

[13] W. Lusky, On weighted spaces of harmonic and holomorphic functions, J. London Math. Soc. 51 (1995), 309-320. MR:1325574 (96d:46020)

[14] W. Lusky, On the isomorphism classes of weighted spaces of harmonic and holomorphic functions. Studia Math. 175 (2006), no. 1, 19-45. MR2261698(2007f:30089)

[15] M.J. Martin, D. Vukotic, Isometries of some classical function spaces among the composition operators. Recent Advances in Operator-Related Function Theory, 133-138, Contemp. Math., 393, Amer. Math. Soc., Providence, RI, 2006. MR2198376 (2006k:47050)

[16] M.J. Martin, D. Vukotic, Isometries of the Bloch space among the composition operators, Bull. London Math. Soc. 39 (2007), 151-155. MR2303530 (2008c:47044)

[17] R.C. Roan, Composition operators on $H^{p}$ with dense range, Indiana Univ. Math. J. 27 (1978), no. 1, 159-162. MR0473905 (57:13564)

[18] D. Sarason, Weak-star generators of $H^{\infty}$, Pacific J. Math. 17 (1966), 519-528. MR 0211269 $(35: 2151)$

[19] J.H. Shapiro, Composition Operators and Classical Function Theory, Springer-Verlag, 1993. MR.1237406 (94k:47049)

Instituto Universitario de Matématica Pura y Aplicada iUmPa-UPV, Edificio idi5 (8E), Cubo F, Cuarta Planta, Universidad Politécnica de Valencia, E-46022 Valencia, SPAIN

E-mail address: jbonet@mat.upv.es

Department of Mathematical Sciences, P.O. Box 3000, Fin-90014, University of Oulu, Oulu, Finland

E-mail address: mikael.lindstrom@oulu.fi

Institute of Mathematics, University of Paderborn, D-33095 Paderborn, Germany

E-mail address: lichte@math.uni-paderborn.de 